\title{
PROPAGATION OF SUPPORT AND SINGULARITY FORMATION FOR A CLASS OF 2D QUASILINEAR HYPERBOLIC SYSTEMS
}

\author{
BY \\ M. C. LOPES FILHO AND H. J. NUSSENZVEIG LOPES \\ Departamento de Matematica, IMECC-UNICAMP, Caixa Postal 6065, Campinas, SP 13081-970, \\ Brasil
}

\begin{abstract}
In this paper we consider a class of quasilinear, non-strictly hyperbolic $2 \times 2$ systems in two space dimensions. Our main result is finite speed of propagation of the support of smooth solutions for these systems. As a consequence, we establish nonexistence of global smooth solutions for a class of sufficiently large, smooth initial data. The nonexistence result applies to systems in conservation form, which satisfy a convexity condition on the fluxes. We apply the nonexistence result to a prototype example, obtaining an upper bound on the lifespan of smooth solutions with small amplitude initial data. We exhibit explicit smooth solutions for this example, obtaining the same upper bound on the lifespan and illustrating loss of smoothness through blow-up and through shock formation.

Consider a quasilinear, hyperbolic system of differential equations of the form:

$$
\left\{\begin{array}{l}
U_{t}+A(U) U_{x}+B(U) U_{y}=0 \\
U(x, 0)=U_{0}(x)
\end{array}\right.
$$

where $U(x, y, t)=(u(x, y, t), v(x, y, t))$ is the state vector and $A(\cdot), B(\cdot)$ are smooth, $2 \times 2$ matrix-valued functions of the state vector. Hyperbolicity means that the matrix $C(\xi, U)=\xi_{1} A(U)+\xi_{2} B(U)$ has real eigenvalues for any $U$ in state space and for any $\xi=\left(\xi_{1}, \xi_{2}\right) \in S^{1}$.

A partially aligned state $U_{0}$ for the system above is a state such that $A\left(U_{0}\right)$ and $B\left(U_{0}\right)$ have a common eigenvector. A partially aligned system is one for which all states are partially aligned. This class of systems was introduced by the authors in [8], along with some basic properties and a classification of different kinds of partial alignment. In this article we will use the results and terminology from [8]. An earlier version of the results contained here was announced in [10].
\end{abstract}

Received July 1, 1996.

1991 Mathematics Subject Classification. Primary 35L40, 35L67, 35L80, 35L65.

Key words and phrases. Propagation of support, lifespan of classical solutions, partially aligned systems.

The first author's research was supported in part by CNPq Grant \#300962/91-6.

The second author's research was supported in part by CNPq Grant \#300158/93-9.

E-mail address: mlopes@ime.unicamp.br

E-mail address: hlopes@ime.unicamp.br 
We highlight three issues concerning partially aligned systems:

1. The characteristic wavefields are two families of curves in physical space, one of which carries a Riemann invariant. (By "characteristic curves", here and in the remainder of this paper, we mean curves along which part of the system reduces to a family of ODEs.) In other words, the basic geometry of partially aligned systems is much simpler than strictly hyperbolic systems and, more importantly, very close to the familiar 1-D situation. We may illustrate this by referring the reader to [9], where a compression rate analysis for shock formation was carried out for some partially aligned systems following closely the analogous 1-D work.

2. Partially aligned systems, particularly far from degenerate states called coincident (states where the characteristic curves are tangent), have characteristics with variable multiplicity, in the microlocal sense. At each non-coincident state the equation is strictly hyperbolic in all directions except two opposing directions where the planar wavespeeds coincide. In contrast with 1-D non-strictly hyperbolic systems, where resonance occurs in localized sites in state space, partially aligned systems have a distributed, weak resonance occurring everywhere at once. This makes partially aligned systems hard to treat analytically. In consequence, even local-wellposedness for smooth initial data is open for partially aligned systems; the only well-posedness result in the literature is due to Kajitani [4], who works in Gevrey classes.

3. There is a rich class of examples of partially aligned systems, which includes some natural models in used applications. One instance is the application to multi- $D$ polymer flooding proposed in [2]. We can write this class of examples in two equivalent ways as:

$$
\left\{\begin{array}{l}
S_{t}+\operatorname{div}(f(S, C))=0, \\
(C S)_{t}+\operatorname{div}(C f(S, C))=0,
\end{array}\right.
$$

or

$$
\left\{\begin{array}{l}
u_{t}+\operatorname{div}(u g(u, v))=0, \\
v_{t}+\operatorname{div}(v g(u, v))=0,
\end{array}\right.
$$

where $f$ and $g$ are vector-valued functions in state space. This class of problems is a straightforward generalization of a class of one-dimensional systems with applications in nonlinear elasticity and polymer flooding in two-phase porous media flow and with a large associated literature; the reader may refer to the survey paper [5] and references therein. We call attention to the equation in the second form, with $g(u, v)=(u, v)$. We may write this particular system as:

$$
u_{t}+\operatorname{div}(u \otimes u)=0,
$$

where $u$ represents the velocity vector of a compressible 2-dimensional flow. This equation has been studied previously in the literature from the point of view of Riemann problems [13] and, by the authors, from the point of view of singularity formation [9]. We regard it as a prototype example of a partially aligned system.

This paper is organized as follows: first we prove a theorem on propagation of support for upper-triangular quasilinear systems not in conservation form. This is our main result. The proof is a delicate, albeit elementary, argument in differential topology where the 
geometry of the characteristics plays an essential role. Next we apply this result to obtain a description of the evolution of the "support" of a smooth solution of a partially aligned system in conservation form. By "support" we mean the compact set where the solution is nonconstant. Following this, we add a structural hypothesis on the fluxes and we use the previous result to show nonexistence of global smooth solutions for a class of initial data, constant outside a compact set. The argument used is an adaptation of an exponential averaging argument due to T. Sideris [12]. We then examine the specific case of system (2). For this system we prove that there exists smooth initial data with arbitrarily small $C^{1}$-amplitude, such that the lifespan of a smooth solution is at most inversely proportional to the initial amplitude. Finally, we obtain a sharper result on the lifespan of smooth solutions of system (2), by exhibiting explicit solutions. These solutions are obtained by symmetry reduction, and their lifespan can be computed directly. We illustrate both mechanisms for breakdown of the classical solution, namely blow-up and shock formation.

Consider the initial-value problem for the upper-triangular system:

$$
\left\{\begin{array}{l}
u_{t}+F(u, v) \cdot \nabla u=G(u, v) \cdot \nabla v \\
v_{t}+\Lambda(u, v) \cdot \nabla v=0 \\
(u(x, 0), v(x, 0))=\left(u_{0}(x), v_{0}(x)\right) .
\end{array}\right.
$$

Let $\xi \in S^{1}$ be a fixed unit vector. Define the semiplane:

$$
\Omega_{0} \equiv\left\{x \in \mathbb{R}^{2} \mid x \cdot \xi>0\right\} .
$$

Set $S_{0}=\partial \Omega_{0}$.

We consider a constant state vector $W_{*}=\left(u_{*}, v_{*}\right)$ and assume that the linearization of the system (3) at $W_{*}$ is strictly hyperbolic in the direction $\xi$. Since the system is upper-triangular, this is equivalent to requiring that

$$
\Lambda\left(W_{*}\right) \cdot \xi \neq F\left(W_{*}\right) \cdot \xi
$$

Let $M=\max \left\{\Lambda\left(W_{*}\right) \cdot \xi, F\left(W_{*}\right) \cdot \xi\right\}$. Define

$$
\Omega_{T} \equiv\left\{(x, t) \in \mathbb{R}^{2} \times(0, T) \mid x \cdot \xi>M t\right\}
$$

and

$$
\Omega_{T}^{\varepsilon_{0}} \equiv\left\{(x, t) \in \mathbb{R}^{2} \times[0, T) \mid x \cdot \xi>M t-\varepsilon_{0}\right\}
$$

for some $\varepsilon_{0}>0$.

Let $W(x, t)=(u(x, t), v(x, t))$ be a classical solution of system $(3)$, defined on $\Omega_{T}^{\varepsilon_{0}}$ assumed bounded, together with its first derivatives and with initial data $W(x, 0)=$ $W_{0}(x)=\left(u_{0}(x), v_{0}(x)\right)$.

The basic difficulty behind the theorem on propagation of support is tracking the backwards reachable set of the pair of vector fields $F(W(\cdot))$ and $\Lambda(W(\cdot))$, in the sense of control theory (see [7]). If the vector fields were in involution, Frobenius' Theorem would essentially trivialize the problem by making it one-dimensional. In [8] the authors 
introduced the terminology "Huygens systems" for partially aligned systems whose characteristic fields are in involution a priori. The backwards reachable set of a pair of vector fields not in involution may have a very complicated global geometry.

Theorem 1. If $W_{0}(x) \equiv W_{*}$ in $\Omega_{0}$, then $W(x, t) \equiv W_{*}$ in $\Omega_{T}$.

Proof. We begin by assuming that $W(\cdot, t)$ is defined in the whole plane. Let $\Phi^{\Lambda}=$ $\Phi^{\Lambda}(x ; t)$ and $\Phi^{F}=\Phi^{F}(s ; t)$ be the fluxes associated to the characteristic vector fields $(\Lambda, 1)$ and $(F, 1)$ respectively. These fluxes are global diffeomorphisms for each fixed time $t \in[0, T)$, because the solution $W(x, t)$ is assumed to be $C^{1}$-bounded.

Given a set $\Sigma \subseteq \mathbb{R}^{2} \times\{t=0\}$ we define $\Phi^{\Lambda}(\Sigma)$ as the collection of all forward orbits of the $\Lambda$ characteristic field, emanating from $\Sigma$, for time $t$ running the interval $[0, T)$; similarly for $\Phi^{F}(\Sigma)$.

Set $S^{\Lambda} \equiv \Phi^{\Lambda}\left(S_{0}\right)$ and $S^{F} \equiv \Phi^{F}\left(S_{0}\right)$. These are smooth, embedded submanifolds with boundary $S_{0}$, diffeomorphic to semiplanes and each of them decomposes $\mathbb{R}^{2} \times[0, T)$ into two connected components.

The hypothesis of strict hyperbolicity of the direction $\xi$ implies that $F\left(W_{*}\right) \cdot \xi \neq$ $\Lambda\left(W_{*}\right) \cdot \xi$, which in turn implies that the surfaces $S^{\Lambda}$ and $S^{F}$ are transversal at points on $S_{0}$. We observe that $v(x, t)$ is constant and equal to $v_{*}$ in $\Phi^{\Lambda}\left(\Omega_{0}\right)$. The difficulty is that this is not enough to conclude that $u$ is constant and equal to $u_{*}$ in $\Phi^{\Lambda}\left(\Omega_{0}\right) \cap \Phi^{F}\left(\Omega_{0}\right)$, since backwards $F$-characteristics, emanating from $\Phi^{\Lambda}\left(\Omega_{0}\right) \cap \Phi^{F}\left(\Omega_{0}\right)$ may leave $\Phi^{\Lambda}\left(\Omega_{0}\right)$ and hence pick up some nonzero $\nabla v$. We will divide the proof into two cases, according to the relative position of $F\left(W_{*}\right)$ and $\Lambda\left(W_{*}\right)$.

CAse I. Let us assume that $\Lambda\left(W_{*}\right) \cdot \xi<F\left(W_{*}\right) \cdot \xi$.

Claim 1. Let $p$ be a point on $S_{0}$. There exists a neighborhood $B$ of $p$ in $\mathbb{R}^{2} \times[0, T)$ such that:

(a) $S^{F} \cap B \subseteq \Phi^{\Lambda}\left(\Omega_{0}\right) \cup S_{0}$ and $S^{F} \cap S^{\Lambda} \cap B=S_{0} \cap B$.

(b) $W=W_{*}$ in $B \cap \Phi^{F}\left(\Omega_{0}\right)$ and $S^{F} \cap B$ is a plane segment.

Proof of Claim. Let $\tilde{B}$ be a neighborhood of $p$ such that there exists $z \in \mathbb{R}$ satisfying: For any $(x, t)$ in $\tilde{B}$ we have $\Lambda(W(x, t)) \cdot \xi<z<F(W(x, t)) \cdot \xi$. This implies that the plane segment $\{x \cdot \xi=z t\} \subseteq \mathbb{R}^{2} \times[0, T)$ separates $S^{F}$ and $S^{\Lambda}$ inside $\tilde{B}$. This gives (a) for any sub-neighborhood of $\tilde{B}$. To guarantee (b), choose $B$ an $F$-flow tubular neighborhood of the $F$-characteristic emanating from $p$, truncated at a positive time so that $B \subseteq \tilde{B}$. It is easy to see that (b) holds inside $B$.

Let $\gamma^{p}:[0, T) \rightarrow S^{F}$ be the forward $F$-characteristic emanating from $p$.

Claim 2. If, for every $p \in S_{0}, \gamma^{p}((0, T)) \cap S^{\Lambda}=\varnothing$, then $W \equiv W_{*}$ in $\Omega_{T}$.

Proof of Claim. Let $q \in \Phi^{F}\left(\Omega_{0}\right)$. The backwards $\Lambda$-characteristic from $q$ lies in the same connected component of $\mathbb{R}^{2} \times[0, T) \backslash S^{\Lambda}$ as $S^{F}$. Hence $v=v_{*}$ at $q$, and therefore in $\Phi^{F}\left(\Omega_{0}\right)$. Clearly, the backwards $F$-characteristic emanating from $q$ cannot intercept $S^{F}$. By connectivity, it does not intercept $S^{\Lambda}$ either. Hence it is entirely contained in $\Phi^{\Lambda}\left(\Omega_{0}\right)$. We conclude that $u(q)=u_{*}$. Since $q$ is arbitrary, $W=W_{*}$ in $\Phi^{F}\left(\Omega_{0}\right)$. By continuity, $W=W_{*}$ on $S^{F}$, so that $\Phi^{F}\left(\Omega_{0}\right)=\Omega_{T}$.

Suppose, by contradiction, there exists $p_{0} \in S_{0}$ such that $\gamma^{p_{0}} \cap S^{\Lambda} \neq \varnothing$. Define

$$
t_{0}=\min _{0 \leq t<T}\left\{t \mid \gamma^{p_{0}}(t) \in S^{\Lambda}\right\} .
$$


Observe that $t_{0}>0$ because no intersection occurs inside $B$. Additionally, we note that $\gamma^{p_{0}}\left(\left[0, t_{0}\right]\right)$ is a straight line, parallel to $\left(F\left(W_{*}\right), 1\right)$, since this is an $F$-characteristic that does not cross $S^{\Lambda}$ and starts out in $\Phi^{\Lambda}\left(\Omega_{0}\right) \cup S_{0}$. Define $q_{0}=\gamma^{p_{0}}\left(t_{0}\right) \in S^{\Lambda} \cap S^{F}$.

For $s \in\left(0, t_{0}\right)$ consider the backwards $\Lambda$-characteristic emanating from $\gamma^{p_{0}}(s)$, which we will denote by

$$
\eta_{s}:[0, s] \rightarrow \mathbb{R}^{2} \times[0, s]
$$

with $\eta_{s}(s)=\gamma^{p_{0}}(s)$ and $\eta_{s}(0) \in \mathbb{R}^{2} \times\{t=0\}$. There exists an $\varepsilon>0$ such that for $0 \leq s<\varepsilon$, the whole curve $\eta_{s}$ is contained in $B \cap \Phi^{F}\left(\Omega_{0}\right)$, which is a straight line segment, since $W=W_{*}$ in $B \cap \Phi^{F}\left(\Omega_{0}\right)$.

Claim 3. There exists $\tilde{s} \in\left(0, t_{0}\right)$ such that $\eta_{\tilde{s}}([0, \tilde{s})) \cap S^{F}$ is nonempty.

Proof of Claim. Assume, by contradiction, that $\eta_{s}([0, s)) \cap S^{F}=\varnothing$ for every $s \in$ $\left(0, t_{0}\right)$. It is enough to show that $\eta_{s}([0, s])$, for $0<s<t_{0}$ are straight line segments, parallel to $\left(\Lambda\left(W_{*}\right), 1\right)$. Indeed, if this is the case, the backwards $\Lambda$-characteristic through $q_{0}$ is the line segment $q=q_{0}+\left(s-t_{0}\right)\left(\Lambda\left(W_{*}\right), 1\right)$. This straight line intercepts $\{t=0\}$ at $q=q_{0}-t_{0}\left(\Lambda\left(W_{*}\right), 1\right)$, which does not belong to $S_{0}$ due to the transversality hypothesis $\Lambda\left(W_{*}\right) \cdot \xi \neq F\left(W_{*}\right) \cdot \xi$. Therefore, $q_{0}$ cannot belong to $S^{\Lambda}$, a contradiction.

It remains to be proven that all of the $\eta_{s}$ are straight line segments.

Fix $t<t_{0}$. Observe that there exists an $F$-flow tubular neighborhood $U$ of $\gamma^{p_{0}}([0, t])$ such that $U \cap S^{\Lambda} \subseteq B$, since both $S^{\Lambda}$ and $\gamma^{p_{0}}([0, t])$ are closed sets whose only intersection occurs at $p_{0}$. We argue that this implies that $u=u_{*}$ in $U \cap \Phi^{F}\left(\Omega_{0}\right)$. This is true because the only way for $u$ not to be $u_{*}$ is for a backwards $F$-characteristic to intercept $S^{\Lambda}$. These backwards $F$-characteristics inside $U$ only intercept $S^{\Lambda}$ inside $B$, and hence they must all lie in $\left(\Phi^{F}\left(\Omega_{0}\right)\right)^{c}$. We conclude that

$$
u=u_{*} \quad \text { in } U \cap \Phi^{F}\left(\Omega_{0}\right)
$$

Of course, for $0<s<t_{0}, \eta_{s}([0, s]) \subseteq \Phi^{\Lambda}\left(\Omega_{0}\right)$; so $v=v_{*}$ on these curves. It is enough to prove that $u=u_{*}$ there as well. Consider the set $D \equiv\left\{s \in\left(0, t_{0}\right) \mid \exists q \in \eta_{s}([0, s))\right.$ with $\left.u(q) \neq u_{*}\right\}$. We want to show that $D$ is empty. Suppose it is not empty. Let $s_{1}=\inf D$. Clearly, $s_{1}>0$ by the observation preceding the statement of Claim 3 .

By continuity $u=u_{*}$ on $\eta_{s_{1}}\left(\left[0, s_{1}\right)\right)$. Therefore there must be a sequence of points $p_{n}$ on $\eta_{s^{n}}\left(\left[0, s^{n}\right)\right)$, for $s^{n} \searrow s_{1}$ with $u\left(p_{n}\right) \neq u_{*}$. Also, $p_{n} \in \Phi^{F}\left(\Omega_{0}\right)$ because $s_{1}<t_{0}$. Hence the backwards $F$-characteristic emanating from $p_{n}, \gamma^{p_{n}}$, intercepts $S^{\Lambda}$. There is clearly a converging subsequence of $\left\{p_{n}\right\}$ with limit $\bar{p} \in \eta_{s_{1}}\left(\left[0, s_{1}\right]\right)$. We cannot have $\bar{p}=\eta_{s_{1}}\left(s_{1}\right)=\gamma^{p_{0}}\left(s_{1}\right)$ due to (5). Thus $\bar{p}=\eta_{s_{1}}(\bar{s})$ for some $0 \leq \bar{s}<s_{1}$. Consider the backwards $F$-characteristic emanating from $\bar{p}, \gamma^{\bar{p}}$. This $F$-characteristic reduces to the point $\eta_{s_{1}}(0)$ if $\bar{s}=0$. By uniqueness of solutions of ODEs, $\gamma^{\bar{p}}([0, \bar{s}])$ has to be the straight line, parallel to $\gamma^{p_{0}}$ through $\bar{p}$, contained in the triangle formed by the straight line characteristics $\eta_{s}$ for $0 \leq s \leq s_{1}$. Hence $\gamma^{\bar{p}}([0, \bar{s}]) \cap S^{\Lambda}=\varnothing$. There exists an $F$-flow tubular neighborhood of $\gamma^{\bar{p}}$ disjoint from $S^{\Lambda}$. We conclude that $u=u_{*}$ in this neighborhood, contradicting the fact that $\bar{p}$ is accumulated by a subsequence of $p_{n}$. Therefore, $D$ is empty. This implies that the $\eta_{s}$ are straight line segments, which concludes the proof of Claim 3. 
Let

$$
s_{0} \equiv \inf _{0 \leq s \leq t_{0}}\left\{s \mid \eta_{s}([0, s)) \cap S^{F} \neq \varnothing\right\}
$$

By Claim 3, the set considered in the definition of $s_{0}$ is nonempty. Clearly, $s_{0} \geq \varepsilon>0$, where $\varepsilon$ was defined so that $\eta_{s}$ is contained in $B \cap \Phi^{F}\left(\Omega_{0}\right)$ for $0 \leq s<\varepsilon$.

There are two cases to consider: Either the infimum in the definition of $s_{0}$ is assumed, so that $\eta_{s_{0}}\left(\left[0, s_{0}\right)\right) \cap S^{F}$ is nonempty, or $s_{0}<t_{0}$ and $\eta_{s_{0}}\left(\left[0, s_{0}\right)\right) \cap S^{F}=\varnothing$, and $\eta_{s_{0}}$ is accumulated by $\Lambda$-characteristics that do intercept $S^{F}$.

It is easy to see that the first case leads to a contradiction. In fact, there exists $\bar{q} \in \eta_{s_{0}}\left(\left[0, s_{0}\right)\right) \cap S^{F}$. A consequence of the proof of Claim 3 is that for $s<s_{0}$, the $\eta_{s}$ are parallel straight lines. It follows that the backwards $F$-characteristic through $\bar{q}$ is the straight line parallel to $\gamma^{p_{0}}$, contained in the triangle filled by the straight line characteristics $\eta_{s}$, for $0 \leq s \leq s_{0}$. Clearly this $F$-characteristic does not intercept the plane $\{t=0\}$ in $S_{0}$, contradicting the fact that $\bar{q} \in S^{F}$.

Let us now address the second case, where $s_{0}<t_{0}$. There exists a sequence of points $\left\{q_{n}\right\}$ and numbers $s^{n} \searrow s_{0}$ such that $q_{n} \in \eta_{s^{n}}\left(\left[0, s^{n}\right)\right) \cap S^{F}$. The $q_{n}$ form a bounded sequence; so there is a converging subsequence to some point $\bar{q} \in \eta_{s_{0}}\left(\left[0, s_{0}\right]\right) \cap S^{F}$. Since $\eta_{s_{0}}(0) \notin S_{0}$, and since $\eta_{s_{0}}\left(\left[0, s_{0}\right)\right) \cap S^{F}=\varnothing$ we must have $\bar{q}=\gamma^{p_{0}}\left(s_{0}\right)$.

First observe that the straight line segment $\eta_{s_{0}}\left(\left[0, s_{0}\right]\right)$ is tangent to $S^{F}$ at $\gamma^{p_{0}}\left(s_{0}\right)$. To see this approximate (in the sense of $C^{1}$ ) $\eta_{s_{0}}$ by the neighboring $\Lambda$-characteristics $\eta_{s^{n}}$, each of which intersects $S^{F}$ at two distinct points, $\gamma^{p_{0}}\left(s^{n}\right)$ and $q_{n}$, both near $\bar{q}$. The tangency follows from using a "straightening out" diffeomorphism on $S^{F}$, close to $\gamma^{p_{0}}\left(s_{0}\right)$, and then applying the mean value theorem. However, the tangent plane to $S^{F}$ at $\gamma^{p_{0}}\left(s_{0}\right)$ is parallel to the tangent plane to $S^{F}$ at $p_{0}$.

To prove this last statement, define $t_{0}(p)$, for $p \in S_{0}$, analogously to (4). It is easy to see that $t_{0}=t_{0}(p)$ is a lower semicontinuous function. Hence, the portion of $S^{F}$ under the graph of $t_{0}$ is open, in $S^{F}$, and is contained in a plane. Since $s_{0}<t_{0}\left(p_{0}\right)$ the tangent plane to $S^{F}$ at $\gamma^{p_{0}}\left(s_{0}\right)$ is what we wished.

The fact that $\eta_{s_{0}}\left(\left[0, s_{0}\right]\right)$ is tangent to $S^{F}$ at $\gamma^{p_{0}}\left(s_{0}\right)$ and the fact that the tangent plane to $S^{F}$ at $\gamma^{p_{0}}\left(s_{0}\right)$ is as above are mutually contradictory, by transversality.

Therefore, $\gamma^{p_{0}} \cap S^{\Lambda}=\varnothing$ and, by Claim 2, the proof of Case I is concluded.

CASE II. Let us now consider the other case, where $\Lambda\left(W_{*}\right) \cdot \xi>F\left(W_{*}\right) \cdot \xi$. An argument similar to the one above can be used. However, there are certain essential differences due to the intrinsic asymmetry between the $F$ - and the $\Lambda$-characteristic fields. When we refer to the analogous statement or proof here, we mean the corresponding statement or proof contained in Case I, with $F$ and $\Lambda$ exchanged.

The analogous statement to Claim 1 holds, but the proof has to be modified. The choice of the neighborhood $\tilde{B}$ is made in the analogous way, but $B$ is still chosen to be an $F$-flow tubular neighborhood. To prove part (b), one needs to observe that the $(F, 1)$ vector field is transversal to $S^{\Lambda}$ inside $B$, outgoing with respect to $\Phi^{\Lambda}\left(\Omega_{0}\right)$. Hence (b) follows.

We introduce the $\Lambda$-characteristic $\gamma^{p}$ and the backwards $F$-characteristics $\eta_{s}$ in the analogous fashion. Claim 2 and Claim 3 have to be substituted by the following statement: 
Claim 4. If for any $p \in S_{0}$ and any $s \in(0, T), \eta_{s}([0, s))$ does not intercept $S^{\Lambda}$, then $W \equiv W_{*}$ in $\Omega_{T}$.

Proof of Claim. First observe that, under this hypothesis, there is no intersection between $\gamma^{p}$ and $S^{F}$. To see that, assume otherwise. Note that for times $s$ prior to the first positive intersection time of $\gamma^{p}$ and $S^{F}, t_{0}=t_{0}(p)$ (it exists because no intersection occurs inside $B)$, both $\eta_{s}([0, s])$ and $\gamma^{p}([0, s])$ are straight line segments, parallel to $\left(F\left(W_{*}\right), 1\right)$ and $\left(\Lambda\left(W_{*}\right), 1\right)$ respectively. Indeed, the $\eta_{s}$ are contained in $\Phi^{\Lambda}\left(\Omega_{0}\right)$; hence $v=v_{*}$ on $\eta_{s}$. Also, since $s<t_{0}, \eta_{s}$ is contained in $\Phi^{F}\left(\Omega_{0}\right)$ and $u=u_{*}$ there. Clearly this implies that $\gamma^{p}$ is also a straight line. Moreover, extending this argument by continuity until $t_{0}$ we conclude, due to transversality, that no first intersection can occur.

By connectivity and the argument above, $\Phi^{\Lambda}\left(\Omega_{0}\right)=\Omega_{T}$. The claim is proved once we show that $u=u_{*}$ in $\Phi^{\Lambda}\left(\Omega_{0}\right)$. On $S^{\Lambda}$ all backwards $F$-characteristics lead into $\Phi^{\Lambda}\left(\Omega_{0}\right)$, as we have seen. Therefore no backwards $F$-characteristic emanating from points in $\Omega_{T}$ can intercept $S^{\Lambda}$. Hence $u=u_{*}$ in $\Omega_{T}$.

Let us now assume, by contradiction, that there exists $p_{0} \in S_{0}$ such that, for some $0<s<T$, the backwards $F$-characteristic emanating from $\gamma^{p_{0}}, \eta_{s}([0, s])$, intercepts $S^{\Lambda}$. Define $s_{0}$ analogously to Case I. Note that $s_{0}>0$ as in Case I. Also, a consequence of the proof of Claim 4 is that $\eta_{s}([0, s)) \subseteq \Phi^{F}\left(\Omega_{0}\right)$ for $0<s<s_{0}$.

We have two possibilities, as before: either $s_{0}$ is a minimum or $s_{0}$ is an infimum, which is not assumed. We dispose of the first alternative in a way analogous to what we did in Case I. While examining the second alternative, we begin by noticing that the $F$-characteristic $\eta_{s_{0}}$ is tangent to $S^{\Lambda}$ at $\gamma^{p_{0}}\left(s_{0}\right)$ in the same way as before. This leads to a contradiction if we can show that the tangent plane to $S^{\Lambda}$ at $\gamma^{p_{0}}\left(s_{0}\right)$ is parallel to the tangent plane to $S^{\Lambda}$ at $p_{0}$. This is more delicate than in Case I.

To prove this last statement, we must consider $s_{0}=s_{0}(p)$ defined analogously to $(6)$, for any $p \in S_{0}$.

Claim 5. The function $s_{0}=s_{0}(p)$ is lower semicontinuous.

Proof of Claim. We argue by contradiction. Let $\tilde{s}_{0} \equiv \liminf s_{0}(p)$, as $p \rightarrow p_{0}$ and assume $\tilde{s}_{0}<s_{0}$. Let $p_{n}$ be a sequence of points on $S_{0}, p_{n} \rightarrow p_{0}$, with $s_{0}\left(p_{n}\right) \rightarrow \tilde{s}_{0}$. By approximating $F$-characteristics similarly to what was done in Case I and by using the fact that $\tilde{s}_{0}<s_{0}$ we conclude that $\eta_{\tilde{s}_{0}}$ is tangent to $S^{\Lambda}$ at $\gamma^{p_{0}}\left(\tilde{s}_{0}\right)$. Now, $\gamma^{p_{0}}\left(\tilde{s}_{0}\right)$ is at the boundary of an open region in $S^{\Lambda}$ ruled by the straight lines $p+\tau\left(\Lambda\left(W_{*}\right), 1\right)$, with $p \in S_{0}$, near $p_{0}$. Hence the tangent plane to $S^{\Lambda}$ at $\gamma^{p_{0}}\left(\tilde{s}_{0}\right)$ is parallel to the tangent plane to $S^{\Lambda}$ at $p_{0}$. By transversality, this contradicts the tangency of $\eta_{\tilde{s}_{0}}$. Therefore $\tilde{s}_{0} \geq s_{0}$; hence $s_{0}=s_{0}(p)$ is lower semicontinuous.

Claim 5 implies that the tangent plane to $S^{\Lambda}$ at $\gamma\left(s_{0}\right)$ is a translation of the tangent plane at $p_{0}$. With this we obtain a contradiction, concluding the proof of Case II.

Finally we indicate how to adapt the proof above to the local case, where $W$ is defined in $\Omega_{T}^{\varepsilon_{0}}$ only. Take $\Sigma=\left\{(x, t) \in \Omega_{T}^{\varepsilon_{0}} \mid x \cdot \xi=-\varepsilon_{0} / 2+M t\right\}$. In both cases of the proof above, if $p \in S_{0}$, consider $t_{*}=t_{*}(p)$ to be the first intersection of the characteristic $\gamma^{p}$ with $\Sigma$ (again, a lower semicontinuous function of $p$ ) and substitute $t_{0}$ in the proof by the minimum of its previous value as defined and $t_{*}$. The whole argument follows with few minor changes. 
We point out the contrast between the proof above, basically geometric in content, and the proof of the analogous result, for symmetrizable, strictly hyperbolic systems (see [12]), which relies on energy estimates.

REMARK. It was pointed out to the authors by T. Sideris that the result in Theorem 1 can be obtained by standard energy estimates if the solution is assumed to be $C^{2}$ rather than only $C^{1}$. In addition, it is necessary to assume that the solution $W$ is an $H^{1}\left(\mathbb{R}^{2}\right)$-perturbation of the constant state $W_{*}$. We outline the argument:

Linearize the equations (3) about $W_{*}$, by writing $W=(U, V)+W_{*}$. Perform energy estimates for the $V$ equation and its spatial gradient. Since the $V$ equation does not involve the gradient of $U$, crude estimates and Gronwall's lemma yield an estimate of the form:

$$
\int_{\{x \cdot \xi>M t\}}\left[V^{2}(x, t)+|\nabla V(x, t)|^{2}\right] d x \leq C_{T} \int_{0}^{t} \int_{\{x \cdot \xi>M t\}} U^{2}(x, s) d x d s,
$$

for $0 \leq t \leq T$. The constant $C_{T}$ depends on $T$ and on the $C^{2}$-norm of $(U, V)$ in $\Omega_{T}$. Next, perform energy estimates for the $U$ equation, using the estimate above to get:

$$
\frac{d}{d t} \int_{\{x \cdot \xi>M t\}} U^{2} d x \leq C_{T}\left(\int_{\{x \cdot \xi>M t\}} U^{2} d x+\int_{0}^{t} \int_{\{x \cdot \xi>M t\}} U^{2}(x, s) d x d s\right) .
$$

Use a second-order version of Gronwall's lemma to the effect that, if $h=h(s) \geq 0$, $h(0)=h^{\prime}(0)=0$ and $h^{\prime \prime} \leq C\left(h^{\prime}+h\right)$, then $h \equiv 0$, to conclude the argument.

Our objective now is to consider partially aligned systems in conservation form. Let $W(x, t)=\left(u^{1}(x, t), u^{2}(x, t)\right)$ be a $C^{1}$ solution of the system below, which is $C^{1}$-bounded:

$$
\left\{\begin{array}{l}
u_{t}^{1}+\operatorname{div}\left(\vec{f}_{1}\left(u^{1}, u^{2}\right)\right)=0 \\
u_{t}^{2}+\operatorname{div}\left(\vec{f}_{2}\left(u^{1}, u^{2}\right)\right)=0 \\
\left(u^{1}(x, 0), u^{2}(x, 0)\right)=\left(u_{0}^{1}(x), u_{0}^{2}(x)\right) .
\end{array}\right.
$$

Here, $\vec{f}_{1}=\left(f_{11}, f_{12}\right)$ and $\overrightarrow{f_{2}}=\left(f_{21}, f_{22}\right)$ are smooth functions defined in an open set $\Omega \subseteq \mathbb{R}^{2}$. Also, the solution $W=W(x, t)$ is assumed to be defined in $\mathbb{R}^{2} \times[0, T)$ with values in $\Omega$. Let $W_{*}=\left(u_{*}^{1}, u_{*}^{2}\right)$ be a state such that there exists a neighborhood $B \subseteq \Omega$ of $W_{*}$ where system (7) is partially aligned, non-coincident at $W_{*}$.

We will denote by $\Lambda_{*}$ and $F_{*}$ the characteristic directions at $W_{*}$.

TheOREM 2. Assume that the initial data $W_{0}=W(x, 0)$ is constant and equal to $W_{*}$ in the complement of a ball $B_{R}(0)$, for some $R>0$. Then $W=W(x, t)$ is constant and equal to $W_{*}$ in the complement of the set:

$$
E(t) \equiv \text { convex hull }\left\{B_{R}\left(\Lambda_{*} t\right) \cup B_{R}\left(F_{*} t\right)\right\} .
$$

Proof. Let $p$ be a point in $\partial B_{R}(0)$ and $\xi=p /|p|$ the unit outwards normal to $\partial B_{R}(0)$ at $p$. There are two (antipodal) points in $\partial B_{R}(0)$ for which the corresponding normal vectors are directions of failure of strict hyperbolicity for system (7), linearized at $W_{*}$. We assume that $p$ is not one of these two points and we begin the proof by showing that Theorem 1 still applies in this case, so that if $M=\max \left\{\Lambda_{*} \cdot \xi, F_{*} \cdot \xi\right\}$, then $W \equiv W_{*}$ in

$$
\Omega_{T} \equiv\left\{(x, t) \in \mathbb{R}^{2} \times(0, T) \mid(x-p) \cdot \xi \geq M t\right\} .
$$


Let $\Omega_{0} \equiv\left\{x \in \mathbb{R}^{2} \mid(x-p) \cdot \xi \geq 0\right\}$. To see that Theorem 1 still applies, we must first put the system in form (3), which can only be done locally. Since system (7) is partially aligned in $B$, locally there exists a Riemann invariant that allows us to put (7) into the form (3), see [8]. Let $B_{0} \subseteq B$ denote a neighborhood of $W_{*}$ where one such Riemann invariant is defined. Since the solution $W(x, t)$ is assumed to be uniformly $C^{1}$-bounded, there exists a positive $\delta$ such that, at any point $q \in \mathbb{R}^{2} \times[0, T)$ where $W(q)=W_{*}$ we have: $W(B(q ; \delta)) \subseteq B_{0}$. Therefore there exists an $s>0$ such that the open set

$$
U_{s} \equiv\left\{(x, t) \in \mathbb{R}^{2} \times(0, s) \mid(x-p) \cdot \xi>-s+M t\right\}
$$

is contained in the union $\bigcup_{q \in \bar{\Omega}_{0}} B(q ; \delta)$. We now invoke Theorem 1 to show that $W \equiv W_{*}$ in $\Omega_{T} \cap\left(\mathbb{R}^{2} \times(0, s)\right)$. We can repeat the argument above, since $s$ above depends on $\delta$, which depends only on $B_{0}$. Hence, we can advance the result up to time $2 s$ and so forth. Thus we obtain $W \equiv W_{*}$ in $\Omega_{T}$.

Finally, we allow $p$ to vary over all points in $\partial B_{R}(0)$ where it is allowed, i.e., for all $p$ such that $p \cdot\left(F_{*}-W_{*}\right) \neq 0$. We conclude that

$$
\operatorname{supp}\left(W-W_{*}\right) \subseteq \bigcap_{p \in \partial B_{R}(0), p \cdot\left(F_{*}-W_{*}\right) \neq 0} \Omega_{T}(p)^{c} .
$$

It is a simple exercise to characterize this set as described in the statement.

The following result is a corollary of Theorem 2, which will be important in the proof of the nonexistence of global smooth solutions of system (7).

Lemma 3. Fix $R>0$ and $\xi \in S^{1}$. Consider $E(t)$ the time-variable domain in $\mathbb{R}^{2}$ in the statement of Theorem 2. Define

$$
e(t) \equiv \int_{E(t)} e^{\xi \cdot x} d x
$$

Then there exists $\sigma \in \mathbb{R}$ such that

$$
\frac{d}{d t}(\log e(t)) \leq \sigma
$$

Proof. Choose an orthogonal matrix, with positive determinant, such that

$$
\begin{aligned}
T\left[\begin{array}{l}
1 \\
0
\end{array}\right] & =\frac{\Lambda_{*}-F_{*}}{\left\|\Lambda_{*}-F_{*}\right\|}, \\
T\left[\begin{array}{l}
0 \\
1
\end{array}\right] & =\frac{\left(\Lambda_{*}-F_{*}\right)^{\perp}}{\left\|\Lambda_{*}-F_{*}\right\|} .
\end{aligned}
$$

Consider the change of variables $(x, y) \mapsto\left(\eta_{1}, \eta_{2}\right)$ given by

$$
\left[\begin{array}{l}
\eta_{1} \\
\eta_{2}
\end{array}\right]=T^{-1}\left(\left[\begin{array}{l}
x \\
y
\end{array}\right]-\frac{\Lambda_{*}+F_{*}}{2} t\right) .
$$

Then, in these new variables we write

$$
e(t)=\int_{-R}^{R} \int_{-t \frac{\left\|\Lambda_{*}-F_{*}\right\|}{2}-\sqrt{R^{2}-\eta_{2}^{2}}}^{t \frac{\left\|\Lambda_{*}-F_{*}\right\|}{2}+\sqrt{R^{2}-\eta_{2}^{2}}}\left\{\exp \left\langle\xi, \frac{\Lambda_{*}+F_{*}}{2} t+T\left[\begin{array}{l}
\eta_{1} \\
\eta_{2}
\end{array}\right]\right\rangle\right\} d \eta_{1} d \eta_{2} .
$$

Integrate in $\eta_{1}$ and estimate directly $d / d t(\log e(t))=e^{\prime}(t) / e(t)$ to obtain the result. 
Definition 4. Let $\xi \in S^{1}$. We call $\xi$ a direction of convexity for system (7) if there exists $i \in\{1,2\}$ and a convex function $\phi: \mathbb{R} \rightarrow \mathbb{R}$, with

$$
\int_{\lambda}^{\infty} \frac{d s}{\phi(s)}<\infty, \quad \text { for } \lambda \text { sufficiently large }
$$

such that

$$
\xi \cdot \vec{f}_{i} \geq \phi\left(u^{i}\right) .
$$

We will use this definition to show nonexistence of a global smooth solution to partially aligned systems in certain situations. The basic argument is due to T. Sideris (see [12]). In what follows, as in Lemma 3, we denote $e(t)=\int_{E(t)} e^{\xi \cdot x} d x$, where $E(t)$ was defined in Theorem 2.

Corollary 5 . Let $W_{*}$ be a non-coincident state for system (7) and suppose that (7) is partially aligned in a neighborhood of $W_{*}$. Assume that $W_{0}-W_{*}$ is $C^{1}$ and has compact support. Let $\xi=\left(\xi_{1}, \xi_{2}\right)$ be a direction of convexity for (7) corresponding to the unknown $u^{i}$. There exists a constant $C=C\left(\xi, W_{*}\right) \in \mathbb{R}$ such that if

$$
\int_{\mathbb{R}^{2}}\left(u_{0}^{i}(x)-u_{*}^{i}\right) e^{\xi \cdot x} d x>C
$$

then there are no global $C^{1}$ solutions of (7) with initial data $W_{0}$.

Proof. We proceed by contradiction. Suppose that there exists a $C^{1}$ solution of (7) defined on $\mathbb{R}^{2} \times[0, \infty)$. Without loss of generality we may assume that $W_{*}=(0,0)$ and that $\left.\left(\xi \cdot \vec{f}_{i}\right)\right|_{(0,0)}=0$. This may change the convex function $\phi$ to another convex function, still satisfying (8), which for convenience, we will continue calling $\phi$. Suppose that the support of the initial data is contained in the ball $B_{R}(0)$. Let $E(t)$ be as in Theorem 2 and define

$$
q(t)=\int_{\mathbb{R}^{2}} u^{i}(x, t) e^{\xi \cdot x} d x .
$$

Let $\xi^{\perp}=\left(-\xi_{2}, \xi_{1}\right)$ and $\bar{f}_{i 1}=\xi \cdot \vec{f}_{i}, \bar{f}_{i 2}=\xi^{\perp} \cdot \vec{f}_{i}$. We calculate

$$
\frac{d q}{d t}=\int_{\mathbb{R}^{2}} u_{t}^{i} e^{\xi \cdot x} d x=-\int_{\mathbb{R}^{2}}\left(\frac{\partial \bar{f}_{i 1}}{\partial \xi}+\frac{\partial \dot{\bar{f}}_{i 2}}{\partial \xi^{\perp}}\right) e^{\xi \cdot x} d x .
$$

Denote a point in $\mathbb{R}^{2}$ by $x=x_{1} \xi+x_{2} \xi^{\perp}$ so that we obtain

$$
\begin{aligned}
(9) & =-\int_{\mathbb{R}^{2}}\left(\frac{\partial \bar{f}_{i 1}}{\partial x_{1}}+\frac{\partial \bar{f}_{i 2}}{\partial x_{2}}\right) e^{x_{1}} d x_{1} d x_{2}=-\int_{\mathbb{R}^{2}} \frac{\partial \bar{f}_{i 1}}{\partial x_{1}} e^{x_{1}} d x_{1} d x_{2} \\
& =\int_{\mathbb{R}^{2}} \bar{f}_{i 1}\left(u^{1}, u^{2}\right) e^{x_{1}} d x_{1} d x_{2} \geq e(t) \int_{\mathbb{R}^{2}} \phi\left(u^{1}\right) \frac{e^{x_{1}} d x_{1} d x_{2}}{e(t)} .
\end{aligned}
$$

By Jensen's inequality it follows that

$$
\frac{d q}{d t} \geq e(t) \phi\left(\int_{\mathbb{R}^{2}} u^{i} \frac{e^{x_{1}} d x_{1} d x_{2}}{e(t)}\right)=e(t) \phi(q(t) / e(t)) .
$$

Define $Q(t)=q(t) / e(t)$. We conclude that

$$
\frac{d Q}{d t} \geq \phi(Q)-\left(\frac{d}{d t} \log (e(t))\right) Q
$$


By Lemma 3, the time-dependent coefficient $\left(\frac{d}{d t} \log (e(t))\right)$ is bounded above. Condition (8) on $\phi$ implies $\phi$ is superlinear, so that there exists some constant $s_{0} \geq 0$ such that for any $s \geq s_{0}$, and for all $t \geq 0, \phi(s)-\left(\frac{d}{d t} \log (e(t))\right) s>0$. Hence, if we assume $Q(0) \geq s_{0}$, or in other words, if we take the constant $C$ in the statement of this Theorem to be $C \equiv e(0) s_{0}$, then $Q(t)$ will remain greater than $s_{0}$ for all time. With this observation, using Lemma 3 and integrating the differential inequality in the time interval $[0, T]$, we obtain

$$
T \leq \int_{s_{0}}^{\infty} \frac{d s}{\phi(s)-\sigma s}<\infty
$$

which concludes the proof.

Throughout the rest of this paper we will concentrate our attention on the prototype system (2). We will perform a more detailed analysis of the result obtained, for this special case. In coordinates, it is written as

$$
\left\{\begin{array}{l}
u_{t}+\left(u^{2}\right)_{x}+(u v)_{y}=0 \\
v_{t}+(u v)_{x}+\left(v^{2}\right)_{y}=0
\end{array}\right.
$$

This system is partially aligned at all states, coincident only at $(u, v)=(0,0)$. The authors performed a crude singularity formation analysis of this system in [9]. However, the analysis carried out here provides more definite information. The characteristic vector fields are $\Lambda(u, v)=(2 u, 2 v)$ and $F(u, v)=(u, v)$. We have two directions of convexity, $\xi=(1,0)$ and $\xi=(0,1)$. In fact, this system is invariant under simultaneous rotation of the spatial independent variables and of the state variables by the same angle. This was proved in [6]. This means that the result below, stated and proved for states $u_{*}$ negative and $v_{*}=0$ can be done in any direction in state space, after suitably rotating system (10).

In what follows, our concern is the asymptotic lifespan of smooth solutions with small initial data. Although Corollary 5 applies to sufficiently large initial data, we shall see that it can be used to obtain some information about small initial data problems, in the special case of system (10).

Corollary 6. There exists a constant $K>0$ such that for any $\delta>0$, there exists $u_{*}<0$ and smooth initial data $W_{0}$ for system $(10)$, equal to $\left(u_{*}, 0\right)$ in the complement of the unit disk in $\mathbb{R}^{2}$ and such that $\left\|W_{0}-W_{*}\right\|_{C^{1}} \leq \delta$, for which there is no smooth solution defined up to time $T=K / \delta$.

Proof. Fix $\xi=(1,0)$. We begin by observing that, if $u_{*}<0$, the constant $\sigma$ in Lemma 3 can be chosen to be $u_{*} / 3$. To see this, compute $e(t)$ explicitly,

$$
e(t)=\int_{-1}^{1} \int_{-\sqrt{1-y^{2}}+2 u_{*} t}^{\sqrt{1-y^{2}}+u_{*} t} e^{x} d x d y
$$

Next compute the inner integral, differentiate with respect to time and write the quotient to get

$$
\left(\frac{d}{d t} \log e(t)\right)=u_{*} \frac{\int_{-1}^{1} e^{\sqrt{1-y^{2}}} d y-2 e^{u_{*} t} \int_{-1}^{1} e^{-\sqrt{1-y^{2}}} d y}{\int_{-1}^{1} e^{\sqrt{1-y^{2}}} d y-e^{u_{*} t} \int_{-1}^{1} e^{-\sqrt{1-y^{2}}} d y}<u_{*} / 3
$$


This last estimate follows from observing that $\int_{-1}^{1} \exp \sqrt{1-y^{2}} d y \geq 1+e$ and $\int_{-1}^{1} \exp -\sqrt{1-y^{2}} d y \leq 1+1 / e$.

Now we examine the proof of Corollary 5. The convex function $\phi$ used in the proof can be taken to be

$$
\phi(s)=\left(s+u_{*}\right)^{2}-u_{*}^{2} .
$$

When we determine the constant $C$ in Corollary 5 , we can take it to be $e(0) s_{0}$ where $s_{0}$ is bigger than the largest root of the polynomial $\left(s+u_{*}\right)^{2}-u_{*}^{2}-u_{*} s / 3=0$, which is $-(5 / 3) u_{*}$. We shall take $s_{0}=-2 u_{*}$. After integrating, Corollary 5 says that if

$$
\int_{\sqrt{x^{2}+y^{2}} \leq 1} u_{0} e^{x} d x d y \geq-u_{*} \int_{\sqrt{x^{2}+y^{2}} \leq 1} e^{x} d x d y
$$

then there is no smooth solution for times greater than

$$
T=\frac{C}{u_{*}},
$$

for $C=-(3 \log 6) / 5<0$.

For each $\delta>0$ we must find $u_{*}(\delta)$ and $u_{0}(x, y)$ such that $\left\|u_{0}-u_{*}\right\|_{C^{1}}=\delta$ and $u_{0}=u_{*}$ at the boundary of the unit disk and condition (11) is satisfied. Then we estimate $T$. We obtain the result by taking $u_{0}$ to be a slight modification of a "chinese hat" $\left.u_{0}(x, y)=a-\left(a-u_{*}\right) \sqrt{x^{2}+y^{2}}\right)$ and choosing $a$ so that the $C^{1}$-norm above is $\delta$, then finding a set of $u_{*}<0$ for which condition (11) is valid. The important point is that we can choose $-u_{*} \sim O(\delta)$, which then gives the desired result.

The proof of Corollary 5 gives a very crude estimate on the lifespan of smooth solutions. One may see this by observing that it only uses one of the conservation laws in the system. Hence, it does not take into account the nonlinear interaction between the wavefields and the resonance present in partially aligned systems. A more satisfying result for system (10) can be obtained by exhibiting a family of classical solutions whose breakdown can be described precisely.

Once again, we use the invariance of system (10) under simultaneous rotation of spatial and state variables to perform a symmetry reduction of the system. Let $r=\sqrt{x^{2}+y^{2}}$ and $\theta=\arctan y / x$. We look for rotationally symmetric solutions, assuming the ansatz:

$$
\left\{\begin{array}{l}
u(x, y, t)=a(r, t) \cos \theta-b(r, t) \sin \theta \\
v(x, y, t)=a(r, t) \sin \theta+b(r, t) \cos \theta
\end{array}\right.
$$

Plugging (12) into system (10), we obtain a system of reduced equations for $a$ and $b$ :

$$
\left\{\begin{array}{l}
a_{t}+2 a a_{r}+\frac{1}{r}\left(a^{2}-b^{2}\right)=0, \\
b_{t}+b a_{r}+a b_{r}+\frac{2}{r} a b=0, \\
a(r, 0)=a_{0}(r), \quad b(r, 0)=b_{0}(r), \\
a(0, t)=b(0, t)=0 .
\end{array}\right.
$$

The boundary condition at $r=0$ ensures smoothness of solutions of the form (12) at the origin, at least as long as the solutions of (13) have a well-defined unilateral derivative 
at $r=0$. If $b \equiv 0$ and $a$ satisfies the equation:

$$
\left\{\begin{array}{l}
a_{t}+2 a a_{r}+\frac{1}{r} a^{2}=0, \\
a(r, 0)=a_{0}(r), \quad a(0, t)=0,
\end{array}\right.
$$

then $(u, v)$ given by $u=a \cos \theta$ and $v=a \sin \theta$ satisfies system (10), as long as $a$ is smooth and has a unilateral derivative at $r=0$.

The method of characteristics allows for explicit computation of the solution along characteristics of (14). Let $\alpha>0$ be a Lagrangian marker and let $r=r(\alpha, t)$ be the characteristic curve departing from $\alpha$. Then we have

$$
r(\alpha, t)=\left(3 \alpha^{1 / 2} a_{0}(\alpha) t+\alpha^{3 / 2}\right)^{2 / 3}
$$

and

$$
a(r(\alpha, t), t)=a_{0}(\alpha)\left(\frac{\alpha}{r(\alpha, t)}\right)^{1 / 2}
$$

Therefore, for a given Lagrangian marker $\alpha$, we have a blow-up time:

$$
T_{b u}(\alpha)=-\frac{\alpha}{3 a_{0}(\alpha)}
$$

and a shock formation time (when the map $\alpha \mapsto r(\alpha, t)$ is no longer smoothly invertible):

$$
T_{s f}(\alpha)=-\frac{\alpha}{a_{0}(\alpha)+2 \alpha a_{0}^{\prime}(\alpha)} .
$$

It is easy to see that blow-up cannot happen before shock formation, since $r\left(\alpha, T_{b u}\right)$ is always zero. This means that, at time $T_{b u}$ the characteristic $r(\alpha, t)$ is already crossing the characteristic boundary $r(0, t) \equiv 0$. This means that $\inf _{\alpha} T_{b u}(\alpha) \leq \inf _{\alpha} T_{s f}(\alpha)$. Equality, however, may occur, for example when $a_{0}(\alpha)=-\alpha$, which corresponds to the solution

$$
(u, v)(x, y, t)=-\left(\frac{x}{1-3 t}, \frac{y}{1-3 t}\right) .
$$

There is a particular solution to Burgers' equation, with a similar structure, that is understood as an infinite speed shock wave, not as a blow-up.

Better examples, where the initial data are smooth and have compact support can be obtained, which have no analogue for Burgers' equation. Let $\varphi \in C_{c}^{\infty}([0, \infty))$ be a function such that: $\varphi(0)=1,0 \leq \varphi(\alpha)<1$ for $\alpha>0, \varphi^{\prime}(0)=0$ and $\varphi$ is nonincreasing. Choose $a_{0}(\alpha)=-\alpha \varphi(\alpha)$. For this initial data,

$$
T_{b u}(\alpha)=\frac{1}{3 \varphi(\alpha)} \leq \frac{1}{3 \varphi(\alpha)+2 \alpha \varphi^{\prime}(\alpha)}=T_{s f}(\alpha) .
$$

In this case, shock formation and blow-up occur simultaneously at $T=1 / 3$.

It is much easier to produce examples where shock formation occurs before blow-up. Any negative, smooth, compactly supported function, with support away from $r=0$ develops shocks, which happen before blow-up. Moreover, note that any initial data $a_{0} \geq 0$ such that there exists $\alpha$ with $a_{0}(\alpha)+2 \alpha a_{0}^{\prime}(\alpha)<0$ develops shocks, without ever blowing up.

The examples above complete the analysis begun in [9]. Furthermore, we have obtained the following improvement of Corollary 6 . 
Proposition 7. There exist smooth, compactly supported profiles $\left(u_{0}, v_{0}\right)$ and $T_{0}>0$ such that the problem

$$
\left\{\begin{array}{l}
u_{t}+\left(u^{2}\right)_{x}+(u v)_{y}=0 \\
v_{t}+(u v)_{x}+\left(v^{2}\right)_{y}=0 \\
u(x, y, 0)=\delta u_{0}, \quad v(x, y, 0)=\delta v_{0}
\end{array}\right.
$$

has a classical smooth solution, defined precisely up to time $T_{\delta}=T_{0} / \delta$.

Proof. It is enough to take $\left(u_{0}, v_{0}\right)=a_{0}(r)(\cos \theta, \sin \theta)$, with $a_{0}$ any of the initial data in the examples discussed above. In this case,

$$
T_{0}=\min \left\{\inf _{\alpha} T_{b u}(\alpha), \inf _{\alpha} T_{s f}(\alpha)\right\}
$$

It is obvious that the scaling in the formulas for $T_{s f}$ and $T_{b u}$ obtained above yields the result.

The analysis we have carried out on the reduced system still does not take into account the resonance present in system (10), and is reflected in the failure of strict hyperbolicity of system (13) at $a=0$. The resonance may shorten even further the lifespan of smooth solutions. However, to capture this effect, one must analyze the full system of equations for $a$ and $b,(13)$, from the point of view of lifespan of smooth solutions. This is a difficult problem, beyond the scope of this work.

There is a large body of work on the lifespan of classical solutions for nonlinear hyperbolic problems. We refer the reader to the monography by F. John, [3] and references therein. Among these, we single out the work by M. Rammaha [11] on the formation of singularities in solutions of the $2 \mathrm{D}$ compressible Euler equations for polytropic fluids; this was the first $2 \mathrm{D}$ result with small data. We also mention the more recent work of S. Alinhac, who studied the lifespan of smooth solutions of the compressible, isentropic axisymmetric 2D Euler equations in [1].

It is clear that the lifespan of smooth solutions for hyperbolic equations is determined by the interaction of hyperbolic dispersion, which becomes stronger in higher dimensions, and the nonlinearity. It is to be expected that solutions to strictly hyperbolic problems in two space dimensions have lifespans inversely proportional to the square of the initial amplitudes.

One of the main points of the present work is to establish that partially aligned systems behave as one-dimensional problems with respect to hyperbolic dispersion, naturally yielding lifespans at the most inversely proportional to the initial amplitudes.

Acknowledgments. The authors are grateful to Grey Ercole, Frederico Furtado, Jorge Hounie, and Renato Pedrosa for many helpful comments and discussions. In addition, the authors thank the Instituto de Matematica Pura e Aplicada (IMPA), where a substantial portion of this work was done, for their kind hospitality.

The authors would also like to thank T. Sideris for calling our attention to the work of M. Rammaha [11] and for the observation made in the remark following Theorem 1. 


\section{REFERENCES}

[1] S. Alinhac, Temps de vie des solutions régulières des équations d'Euler compressibles axisymmétriques en dimension deux, Invent. Math. 111, 627-670 (1993)

[2] P. Daripa, J. Glimm, B. Lindquist, and O. McBryan, Polymer Floods: A case study of nonlinear wave analysis and of instability control in tertiary oil recovery, D.O.E. Research and Development Report 03077-275, New York University, November 1986

[3] F. John, Nonlinear wave equations, formation of singularities, A.M.S. University Lecture Series, vol. 2, Amer. Math. Soc., Providence, RI, 1990

[4] K. Kajitani, The Cauchy problem for nonlinear hyperbolic systems, Bull. Sci. Math., $2^{e}$ série, 110, 3-48 (1986)

[5] B. L. Keyfitz, A survey of nonstrictly hyperbolic conservation laws, Lecture Notes in Mathematics, vol. 1270, Springer-Verlag, New York, 1986, pp. 152-162

[6] B. L. Keyfitz and M. C. Filho Lopes, How to use symmetry to find models for multidimensional systems of conservation laws, Lectures in Applied Math. 29, 273-284 (1993)

[7] A. J. Krener and H. Schättler, The structure of small-time reachable sets in low dimensions, SIAM J. Control and Optim. 27, No. 1, 120-147 (1989)

[8] M. C. Lopes Filho and H. J. Nussenzveig Lopes, Multidimensional hyperbolic systems with degenerate characteristic structure, Matemática Contemporanea 8, 225-238 (1995)

[9] M. C. Lopes Filho and H. J. Nussenzveig Lopes, Singularity formation for a system of conservation laws in two space dimensions, J. Math. Anal. Appl. 200, 538-547 (1996)

[10] M. C. Lopes Filho and H. J. Nussenzveig Lopes, Smooth solutions of multidimensional conservation laws, Z.A.M.M., special issue in applied analysis, Proceedings of the III ICIAM, Hamburg, 1996, pp. 141-144

[11] M. A. Rammaha, Formation of singularities in compressible fluids in two-space dimensions, Proc. Amer. Math. Soc. 107, 3, 705-714 (1989)

[12] T. C. Sideris, Formation of singularities in solutions to nonlinear hyperbolic equations, Arch. Rat. Mech. Anal. 86, 4, 369-382 (1984)

[13] D. Tan and T. Zhang, Two-dimensional Riemann problem for a hyperbolic system of conservation laws, Acta Math. Sci. (English edition) 11 4, 369-392 (1991) 